NGU pathogen. Microbiological analysis was required the most frequently for screening and control purposes (340/822, $41.36 \%)$, and in patients with chronic prostatitis (105/822, $12.77 \%$ of all patients). ST pathogens were detected more frequently in patients with urethritis than in patients from other diagnostic groups $(\mathrm{p}<0.01)$, while Ureaplasma urealyticum was detected without statistically significant difference among different diagnostic groups of patients $(p>0.05)$.

Conclusion Urethritis was confirmed in less than $10 \%$ of patients, and the most samples collected in routine of clinical praxis are from patients without urethritis. In patients with urethritis multiplex PCR test can detect etiology quickly and reliable in almost all cases from noninvasive sample.

Disclosure No significant relationships.

\section{P055 LONDON SEXUAL HEALTH PROGRAMME - DEVELOPING INNOVATIVE SOLUTIONS TO OPEN ACCESS SEXUAL HEALTH SERVICES} ${ }^{4}$ Ryan Kinsella*. ${ }^{1}$ City of London Corporation, Sexual Health Team, London, UK; ${ }^{2}$ City of London Corporation, London, UK; ${ }^{3}$ Greater Glasgow and Clyde NHS, Sandyford Initiative, Glasgow, UK; ${ }^{4}$ Preventx, NH, UK

10.1136/sextrans-2019-sti.259

Background Demand for sexual health services is increasing at a time when public funding has reduced. This Programme is a partnership of local government, working with England's National Health Service to improve access to sexual health services. A key part of our vision was to develop an online sexual health triage, home testing and treatment service, which work with a network of over 40 clinics.

Methods In order to achieve this, the Programme undertook widespread engagement across the city. This included a range of activities with service users, for example, online and clinicbased surveys and focus groups with underrepresented groups. Engagement also took place with clinicians, payorst and other stakeholders. Questions were asked to service users about their usage of services and the acceptability of new innovations, including online based self-sampling options. Clinical organisations were asked about the feasibility of online services being part of the clinical pathway. Alongside this engagement project, new governance arrangements, which took the form of legally binding agreements that enabled the parameters of a pan-London service procurement to take place. At the same time, payors agreed to align pricing for activity in clinics, to support system sustainability for both sides.

Results While many respondents said they value being able to go to a sexual health clinic, over half said they would use an online alternative.. With the governance for this partnership finalised: aligned service specifications for both clinic provision and an e-service,could take place for the online service which launched in January '18. The e-service has registered over $120 \mathrm{k}$ users since then

Conclusion The pressure of growing sexual health need on reduced public resource are self evident, doind more of the same was not a viable or sustainable option. With efficiencies brought about through citywide cooperation, robust clinical and commissioner governance structures, access to services in London has been enhanced.

Disclosure No significant relationships.

\section{P056}

THE ACCEPTABILITY OF DIFFERENT HIV TESTING OPTIONS AMONG YOUNG MEN LIVING IN VANCOUVER, CANADA: A QUALITATIVE STUDY

${ }^{1}$ Caroline Mniszak*, ${ }^{1}$ Anna Carson, ${ }^{1}$ Amy Prangnell, ${ }^{2}$ Jean Shoveller, ${ }^{1}$ Rod Knight. ${ }^{1}$ British Columbia Centre on Substance Use, Vancouver, Canada; ${ }^{2}$ University of British Columbia, School of Population and Public Health, Vancouver, Canada

\subsection{6/sextrans-2019-sti.260}

Background In British Columbia (BC), three testing options are available: nominal, non-nominal and anonymous. Little is known, however, about the factors that influence the acceptability of the different testing strategies, particularly among young men, a group with disproportionately low HIV testing rates.

Methods We draw on data from in-depth, semi-structured interviews with 45 young men (18-30) in Vancouver, BC, in order to identify the factors that influence the acceptability of different HIV testing options.

Results Most participants described not being aware that there were options other than nominal testing available in Vancouver. Upon learning about non-nominal and anonymous testing options, participants described seeing the value of non-nominal testing, insofar as it safeguards their privacy while at the same time providing a pathway to HIV-related health care for those who test positive. Many were concerned, however, that anonymous testing would present challenges to treatment and care for those who test positive. Others expressed concerns about the implications for public health not having access to accurate and up-to-date information about the 'state' of the HIV epidemic. Nevertheless, while participants did not tend to describe anonymous testing as something they would opt for in the future, almost all of the participants felt offering anonymous testing as an option is an important strategy to reduce barriers for key groups of young men (e.g., those living in rural communities).

Conclusion Based on our results, offering non-nominal and anonymous HIV testing represents an important step in increasing the accessibility of HIV testing for some groups of young men who found these approaches both acceptable and preferable (e.g., over nominal testing). However, within our sample, participants were not aware that anonymous and nonnominal testing were available. Developing clear and easy-tounderstand communication strategies about the different approaches to testing may enhance opportunities for uptake of these approaches.

Disclosure No significant relationships.

\section{P057 THE BRAZILIAN STRATEGY FOR HIV SELF-TESTS FREE DISTRIBUTION IN PUBLIC HEALTH}

${ }^{1}$ Mariana Villares, ${ }^{2}$ Pâmela Gaspar*, ${ }^{2}$ Alisson Bigolin, ${ }^{2}$ José Neto, ${ }^{2}$ Regina Comparini, ${ }^{2}$ Adele Benzaken, ${ }^{2}$ Gerson Fernando Pereira. ${ }^{1}$ Ministry of Health of Brazil, Department of Surveillance, Prevention and Control of TISS, HIVIAIDS And Viral Hepatites, Brasilia, Brazil: ${ }^{2}$ Ministry of Health of Brazil, Department of Surveillance, Prevention and Control of Sexually Transmitted Infections, HIVIAIDS and Viral Hepatitis, Brasilia, Brazil

\subsection{6/sextrans-2019-sti.261}

Background In 2017 there were 866 thousand PLHIV in Brazil. Among them, $84 \%$ knew their status. Innovative strategies are fundamental to increase access to testing in a country with a continental dimension and a concentrated epidemic. The $\mathrm{MoH}$ acquired 400 thousand HIVST to reach 
undiagnosed people. This paper presents the Brazilian strategy for HIVST distribution in the public health system from January 2019.

Methods Throughout 2018 we analyzed guidelines, studies and policies on HIVST worldwide, conducted a two-month international cooperation with France to learn from country's experience, participated and presented in 2 HIVST international Webex led by WHO and PAHO and, in addition to the results of HIVST projects conducted in Brazil since 2015, held regular stakeholders committee meetings and defined HIVST distribution strategies.

Results The strategy consists of actions that allow access to people that are not reached by health services or that should be tested more frequently, taking the test to the sites of sociability of the key populations and using secondary distribution among peers and sexual partners of people at increased risk. The strategies are: to sexual partnerships or peers of PrEP users; at events and places of sociability of key populations by health teams and NGOs; to sexual partners of prisoners and performance of assisted testing and secondary distribution in health services. The $\mathrm{MoH}$ developed strategies for communication, user support, referral to diagnostic confirmation and follow-up of PLHIV, as well as strategies for monitoring the distribution and use of tests through a post-test questionnaire. Brazilian MoH launched a dedicated HIVST website, including a FAQ session, and there will be extensive training in February 2019.

Conclusion The Brazilian strategy aims to scale-up access to HIV testing. Quarterly monitoring of the strategy will be of paramount importance in measuring results, making the necessary adjustments and evaluating the expansion of the strategy. Disclosure No significant relationships.

\section{\begin{tabular}{|l|l}
\hline P058 & DESIGN AND PERFORMANCE OF THE ALINITY M STI
\end{tabular} ASSAY FOR THE DETECTION OF CT, NG, TV, AND MG}

Kevin Nelson*, Ajith Joseph, Russ Wiesneth, Rachel Lander, Dick Hwang, Chris Jacobson, Frances Lennon, Ketrija Touw, Qingbei Zhang, Joseph Toonen, Alanna Connors, Jinal Patel, Olga Kornfeld, James Lee, Mark Frinder, Shiaolan Ho. Abbott Molecular, Des Plaines, USA

10.1136/sextrans-2019-sti.262

Background The Alinity $\mathrm{m}$ STI assay is an in vitro assay for the qualitative detection of nucleic acids from Chlamydia trachomatis (CT), Neisseria gonorrhoeae (NG), Trichomonas vaginalis, and Mycoplasma genitalium (MG) for use on the automated Alinity $\mathrm{m}$ System. For CT, NG, and TV, the assay may be used to test endocervical swabs, clinician-collected and self-collected vaginal swabs, gynecological specimens in PreservCyt, female urine, and male urine from symptomatic and asymptomatic individuals. For $\mathrm{MG}$, the assay may be used to test endocervical swabs from symptomatic and asymptomatic individuals.

Methods The Alinity m STI assay is designed for the Alinity $\mathrm{m}$ System, a fully automated continuous access analyzer that utilizes magnetic microparticle sample preparation chemistry, unit-dose lyophilized amplification reagents, and ReadiFlex ${ }^{\mathrm{TM}}$ processing logistics to deliver a time-to-first-result of 115 minutes. The assay can be customized to report any combination of CT, NG, TV, or MG from a single test to allow flexibility in the management of laboratory testing. In addition to CT, NG, TV and MG, the assay detects an endogenous human DNA sequence and an exogenous internal control as validity controls for sample adequacy, extraction, and amplification efficiency.

Results Performance characteristics of the Alinity m STI assay were evaluated in a clinical study. Endocervical swabs, vaginal swabs, gynecological specimens in PreservCyt, and urine were collected from 398 females. Urine was collected from 411 males. For each subject, the Alinity specimen type was compared to a matched specimen tested with CE marked assays for CT, NG, TV, and MG. For all analytes, the overall positive percent agreement ranged from $91.4 \%$ to $98.2 \%$ and the overall negative percent agreement ranged from $99.7 \%$ to $100 \%$.

Conclusion The Alinity m STI assay is a sensitive and specific assay for the detection and differentiation of CT, NG, TV, and MG on a state-of-the-art instrument.

Disclosure No significant relationships.

\section{P059 QUANTITATIVE DETECTION OF BACTERIA ASSOCIATED WITH BV IN URINE VERSUS SWAB SAMPLES USING DROPLET DIGITAL PCR}

Deshanta Naicker*. Nelson R Mandela School of Medicine, Clinical Medicine, Durban, South Africa

\subsection{6/sextrans-2019-sti.263}

Background Bacterial vaginosis is the most common vaginal condition found in women of reproductive age. The lack of published data on the detection of BV-associated pathogens from urine, a non-invasive sample, lends novelty to the present study. This study aimed to detect and quantify Gardnerella vaginalis, Prevotella bivia, Atopobium vaginae and Lactobacillus crispatus from urine, as an alternative non-invasive method to vaginal swabs from pregnant women using droplet digital PCR (ddPCR).

Methods A total of $n=100$ DNA samples (50 paired urine and swabs) were tested. The samples were stratified as BV negative and positive using the BD MAX Vaginal panel assay (Becton Dickinson). Total DNA was extracted from urine and swabs using the PureLink Microbiome Kit (ThermoFisher Scientific). Droplet digital PCR was used to determine the absolute quantification of the pathogens using commercially available primer and probe sets. Differences in bacterial load between urine and swab samples were evaluated using Spearman's correlation.

Results In BV positive women, the average copies of Gardnerella quantified was 241598 and 441655 copies/ $\mu$ lin the urine and swab, respectively. Prevotella bivia had a mean of 3459 and 6005 copies/ $/ \mu$, whilst Atopobium vaginae was present at a mean of 51055 and 38454 copies/ $\mu \mathrm{l}$ in urine and swab samples, respectively. The Lactobacillus species was present in the

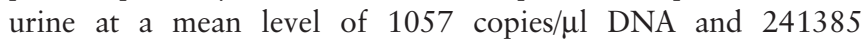
copies/ $\mu \mathrm{l}$ in swabs within BV negative women. A positive correlation between urine and swab samples for all the above mentioned microorganisms was observed $(p<0.0001)$.

Conclusion We observed that urine has the potential to serve as an alternative sample collection method to detect BV-associated bacteria. The data obtained from this pilot study can be used as preliminary data to develop larger studies on this technology. Our future research direction will be to develop ddPCR using urine as a diagnostic test for BV.

Disclosure No significant relationships. 